\title{
THE OPERATOR METHOD FOR ANGULAR MOMENTUM AND SU,
}

\author{
by H. A. M. VAN EEKELEN and TH. W. RUIJGROK
}

Instituut voor Theoretische Fysica, Rijksuniversiteit, Utrecht, Nederland

\section{Synopsis}

It is well known how Schwinger's ${ }^{1}$ ) operator method can be used to construct all representations of the angular momentum operators. We give a brief account of this method and show that it is very convenient for a short derivation of the general Clebsch-Gordan cuefficients. The method is then applied to $\mathrm{SU}_{3}$ and all representations are constructed. Only for a few special cases have we been able to find the ClebschGordan coefficients of $\mathrm{SU}_{3}$.

1. Introduction. It was shown by Schwinger ${ }^{1}$ ) that the algebra of angular momentum simplifies considerably if the operators $L_{x}, L_{y}$ and $L_{z}$ are written in terms of creation and annihilation operators for Bose quanta. Several authors $\left.\left.{ }^{2}\right)^{3}\right)^{4}$ ) have realized that the same method may be applied to $S U_{3}$ and other groups of present interest. In our opinion, however, the operator method has not been exploited sufficiently. It is possible to give a short derivation of some results, of interest to the physicist, using a minimum of abstract mathematical tools. This we will show in the present paper.

The remainder of this section is devoted to the usual derivation of the commutation relations, which the infinitesimal generators of the group $S U_{n}$ must obey. The representations of these generators are then discussed in the following sections. In section 2 the creation and annihilation operators are introduced and it is shown how they can be used to find the representations of the ordinary angular momentum operators. The same is done for $S U_{3}$ in section 4. In the third section we give a short derivation of the ClebschGordan coefficients for the addition of two angular momenta. Some partly solved problems are discussed in the last section.

The group $S U_{n}$ can be defined as the set of all $n$-dimensional unitary matrices $U$, with $\operatorname{det} U=+1$ (unimodular). Every $U$ can be written as

$$
U=\mathrm{e}^{i H},
$$

where $H$ is a hermitian and traceless $n$-dimensional matrix. Since each $H$ is determined by $n^{2}-1$ real numbers it is clear that there exist $n^{2}-1$ linearly independent, hermitian and traccless matriccs $H_{i}\left(i=1, \ldots, n^{2}-1\right)$, 
so that for every $H$

$$
H=\sum_{i=1}^{n^{2}-1} p_{i} H_{i} \quad\left(p_{i} \text { real }\right) .
$$

The matrices $H_{i}$ are the infinitesimal generators of the group. They are not uniquely defined. Their commutation relations, however, are characteristic for the group. The maximum number of generators, that by a unitary transformation can be simultaneously diagonalized is called the rank of the group. For $S U_{n}$ the rank is $(n-1)$. The remaining $\left(n^{2}-n\right)$ generators are usually defined in such a way that all diagonal elements are zero. Above the main diagonal e.g. one could take only one element different from zero and put this element either equal to one or equal to the imaginary unit.

Instead of the generators $H$ and $H^{\prime}$ so obtained, one often uses the linear combinations

$$
H_{ \pm}=\frac{1}{2}\left(H \mp i H^{\prime}\right) .
$$

Because $H_{+}$and $I_{-}$are not hermitian matrices the coefficients $p_{+}$and $p_{-}$in

$$
p_{+} H_{+}+p_{-} H_{-}
$$

must be taken such that (1.4) is hermitian. This implies

$$
p_{+}=p_{-}^{*} \text {. }
$$

Under this condition $H_{+}$and $H_{-}$are again called generators. For $S U_{2}$, which is homomorphic with the group of rotations in a three dimensional real space, the generators are chosen as follows

$$
L_{+}=\left(\begin{array}{ll}
0 & 1 \\
0 & 0
\end{array}\right) ; L_{-}=\left(\begin{array}{ll}
0 & 0 \\
1 & 0
\end{array}\right) ; M=\frac{1}{2}\left(\begin{array}{rr}
1 & 0 \\
0 & -1
\end{array}\right) .
$$

The commutation relations in this case become

$$
\left[L_{+}, L_{-}\right]=2 M \quad \text { and } \quad\left[M, L_{ \pm}\right]= \pm L_{ \pm} .
$$

In the same way we take for the eight generators of $\mathrm{SU}_{3}$ :

$$
\begin{gathered}
M=\frac{1}{2}\left(\begin{array}{rrr}
1 & 0 & 0 \\
0 & -1 & 0 \\
0 & 0 & 0
\end{array}\right), Y=\frac{1}{3}\left(\begin{array}{rrr}
1 & 0 & 0 \\
0 & 1 & 0 \\
0 & 0 & -2
\end{array}\right) \\
L_{+}^{1}=\left(\begin{array}{lll}
0 & 1 & 0 \\
0 & 0 & 0 \\
0 & 0 & 0
\end{array}\right), L_{+}^{2}=\left(\begin{array}{lll}
0 & 0 & 0 \\
0 & 0 & 1 \\
0 & 0 & 0
\end{array}\right), L_{+}^{3}=\left(\begin{array}{lll}
0 & 0 & 0 \\
0 & 0 & 0 \\
1 & 0 & 0
\end{array}\right) \\
L_{-}^{i}=\left(L_{+}^{i}\right)^{*} \quad \text { for } \quad i=1,2,3 .
\end{gathered}
$$

where the asterisk denotes hermitian conjugate. The commutation relations of these matrices are easy to calculate. Introducing the two symbolic matrix 
vectors

$$
s=(M, Y) \quad \text { and } \quad t=\left(M, \frac{3}{4} Y\right)
$$

and the three number vectors

$$
r_{1}=(1,0), \quad r_{2}=\left(-\frac{1}{2}, 1\right), \quad r_{3}=\left(-\frac{1}{2},-1\right)
$$

the commutation relations take the following form

a. $\left[L_{+}^{i}, L_{-}^{i}\right]=2 \boldsymbol{r}_{i} \cdot \boldsymbol{t}$

b. $\left[\boldsymbol{s}, L_{ \pm}^{i}\right]= \pm L_{ \pm}^{i} \boldsymbol{r}_{i}$

c. $\left[L_{+}^{1}, L_{+}^{2}\right]=L_{-}^{3}$ and cyclic

d. $\left[L_{-}^{1}, L_{-}^{2}\right]=-L_{+}^{3}$ and cyclic

$e$. all other commutators equal to zero.

The relations between our generators and those used by De Swart ${ }^{5}$ ) are

$$
L_{ \pm}^{1}=I_{ \pm}, L_{ \pm}^{2}=L_{ \pm}, L_{ \pm}^{3}=K_{\mp}, M=I_{3}, Y=\frac{2}{\sqrt{ } 3} \cdot M .
$$

The operators $L_{ \pm}^{1}$ and $M$ are the generators of a subgroup $S U_{2}$. The operator

$$
L^{2}=L_{+}^{1} L_{-}^{1}+M(M-1)
$$

is usually identified with the total isotopic spin. $Y$ is associated with the hypercharge.

The commutation relations (1.7) and (1.11) are valid not only for the matrices (1.6) and (1.8), but also for the generators, considered as operators of the abstract groups. Since the commutation relations are characteristic for a group the problem of finding the representations of that group reduces to finding the representations of the commutation relations. In the next section this will be done for (1.7). To do the same for (1.11) it is advantageous to detine the following nine operators

$$
\begin{aligned}
& A_{12}=L_{+}^{1} \quad A_{23}=L_{+}^{2} \quad A_{31}=L_{+}^{3} \quad A_{11}=M+\frac{1}{2} Y \\
& A_{21}=L_{-}^{1} \quad A_{32}=L_{-}^{2} \quad A_{13}=L_{-}^{3} \quad A_{22}=-M+\frac{1}{2} Y \text {, } \\
& A_{33}=-Y
\end{aligned}
$$

which satisfy the following relation

$$
A_{11}+A_{22}+A_{33}=0 .
$$

The commutation relations (1.11) can now be written in the condensed form

$$
\left[A_{\mu \nu}, A_{\alpha \beta}\right]=\delta_{\nu \alpha} A_{\mu \beta}-\delta_{\mu \beta} A_{\alpha \nu},
$$

the representations of which will be discussed in section 4 . 
2. Representations of $\mathrm{SU}_{2}$. We want to find the irreducible representations (I.R.'s) of $S U_{2}$ in an explicit and normalized realization, and derive the Clebsch-Gordan coefficients. We introduce the bose operators $a_{1}, a_{1}^{*}, a_{2}, a_{2}^{*}$ which satisfy the usual commutation rules. They operate in the Hilbert space $X$ for which an orthonormal basis is given by

$$
\left|n_{1} n_{2}\right\rangle+\frac{\left(a_{1}^{*}\right)^{n_{1}}\left(a_{2}^{*}\right)^{n_{2}}}{\sqrt{n_{1} ! n_{2} !}}|0\rangle n_{1}, n_{2}=0,1,2 \ldots
$$

We then represent the generators $L_{ \pm}$and $M$ by

$$
\begin{aligned}
& L_{+}=a_{1}^{*} a_{2} \quad L_{-}=a_{2}^{*} a_{1} \\
& M=\frac{1}{2}\left(a_{1}^{*} a_{1}-a_{2}^{*} a_{2}\right) .
\end{aligned}
$$

This is indeed a representation of $S U_{2}$ in $X$ : the commutation relations (1.7) are satisfied. We further introduce

$$
L_{0}=\frac{1}{2}\left(a_{1}^{*} a_{1}+a_{2}^{*} a_{2}\right) .
$$

The operator $L^{2}$, as given by (1.13) can now be written:

$$
L^{2}=L_{0}\left(L_{0}+1\right) \text {. }
$$

We easily reduce our representation in I.R.'s. The subspaces $\{l\}$, defined by

$$
L_{0} \varphi=l_{\varphi} \quad\left(l=0, \frac{1}{2}, 1 \ldots\right)
$$

span the space $X$, and are invariant under the group, because $L_{0}$ commutes with $L_{ \pm}$and $M$ (see (2.2) and (2.1)). The dimension of $\{l\}$ is $2 l+1$, because this is the number of ways in which $2 l$ can be written as the sum of two nonnegative integers. We now take the $2 l+1$ elements

$$
\varphi_{l, m}=\left(L_{-}\right)^{l-m}\left(a_{1}^{\star}\right)^{2 l}|0\rangle=\left(a_{2}^{\star} a_{1}\right)^{l-m}\left(a_{1}^{\star}\right)^{2 l}|0\rangle
$$

with $l-m=0,1, \ldots, 2 l$, or $m=-l, \ldots,+l$. They are orthogonal and elements of $\{l\}$. So they span $\{l\}$, and $\{l\}$ therefore spans an I.R. of the group This I.R. is called $D^{l}$. Abstracting from the explicit representations in the space $X, D^{l}$ is characterized by the eigenvalues $l(l+1)$ of $L^{2}$. The operator $M$ is diagonal on the basis $(2.5)$, with eigenvalues $-l, \ldots,+l$. The normaliization of (2.5) will be given below.

3. Clebsch-Gordan coefficients for $\mathrm{SU}_{2}$. In order to reduce products of two irreducible representations, we work in the product space $X \otimes Y$, which is the direct product of our original space $X$ and a similar one $Y$. In $Y$ we have the Bose operators $b_{1}^{*}, b_{1}, b_{2}^{*}, b_{2}$ and

$$
S_{0}=\frac{1}{2}\left(b_{1}^{*} b_{1}+b_{2}^{*} b_{2}\right) \text {. }
$$

When we put $L_{+}=b_{1}^{*} b_{2}$ as in (2.1), we get a representation of $S U_{2}$ in $Y$, 
which reduces in the same way as the $X$ representation, the I.R. $D^{s}$ being spanned by the subspace $\{s\}$ of $Y$.

In the product space the group is represented by

$$
\begin{aligned}
& L_{+}=a_{1}^{*} a_{2}+b_{1}^{*} b_{2} \quad L_{-}=a_{2}^{*} a_{1}+b_{2}^{*} b_{1} \\
& M=\frac{1}{2}\left(a_{1}^{*} a_{1}-a_{2}^{*} a_{2}+b_{1}^{\star} b_{1}-b_{2}^{\star} b_{2}\right) .
\end{aligned}
$$

For $L^{2}$, as defined by (1.13) we get

$$
L^{2}=\left(L_{0}+S_{0}\right)\left(L_{0}+S_{0}+1\right)-A^{*} A
$$

with

$$
A=a_{1} b_{2}-a_{2} b_{1}
$$

$A$ and $A^{*}$ commute with $L_{ \pm}$and $M$.

We easily see that the subspace $\{l, s\}$ of $X \otimes Y$, defined by

$$
L_{0} \varphi=l \varphi, S_{0} \varphi=s \varphi
$$

is invariant under the group. It transforms according to the representation $D^{l} \otimes D^{s}$. From (3.4) and (3.3) we see that $\{l, s\}$ contains $A^{*}\left\{l-\frac{1}{2}, s-\frac{1}{2}\right\}$. We define another subspace $\Delta^{l, s}$ of $\{l, s\}$ by

a) $\Delta^{l, 8}$ is the orthoplement of $A^{*}\left\{l-\frac{1}{2}, s-\frac{1}{2}\right\}$ in $\{l, s\}$ or, equivalently

b) $\Delta^{l, 8}$ is the subspace of $\{l, s\}$ where $A$ is identically zero.

The equivalence of (3.5) $a$ and $b$ can easily be shown. Take $\varphi \varepsilon\left\{l-\frac{1}{2}, s-\frac{1}{2}\right\}$, and $\psi \varepsilon \Delta^{l, 8}$. If $\Delta^{l, s}$ is defined by (3.5)b, then we have

$$
\left(\psi, A^{*} \varphi\right)=(A \psi, \varphi)=0 . \quad>
$$

If $\Delta^{l, s}$ is defined by (3.5) $a$, then

$$
(A \psi, A \psi)=\left(\psi, A^{*} A \psi\right)=0 . \rightarrow
$$

because $A \psi \varepsilon\left\{l-\frac{1}{2}, s-\frac{1}{2}\right\}$.

From (3.5)b we see that $\Delta^{l, s}$ is an invariant subspace, because $A$ commutes with the group. We now divide $\{l, s\}$ in subspaces (suppose $s \leqslant l$ )

$$
\{l, s\}=A^{*}\left\{l-\frac{1}{2}, s-\frac{1}{2}\right\}+\Delta^{l, s}=\sum_{r=0}^{2 s} A^{*} \Delta^{1-((r / 2), s-(r / 2)} .
$$

All subspaces in (3.6) are invariant, because $A^{*}$ commutes with the group and $\Delta^{l, s}$ is invariant. Further we have from (3.2)

$$
L^{2} \psi=(l+s)(l+s+1) \psi
$$

for $\psi \varepsilon \Delta^{l, s}$.

We now calculate the dimension of $\Delta^{l, 8}$. Take $\psi_{1}, \psi_{2} \varepsilon \Delta^{l, s}$. Using the bose 
commutation rules we have

$$
\left[A, A^{\star r}\right]=A^{* r} r\left(2 L_{0}+2 S_{0}+r+1\right) .
$$

From this we get

$$
\left(A^{*} r \psi_{1}, A^{*}{ }^{*} \psi_{2}\right)=r ! \frac{(2 l+2 s+r+1) !}{(2 l+2 s+1) !}\left(\psi_{1}, \psi_{2}\right) .
$$

The factorials at the right hand side are always positive, so we conclude that the dimension of $A^{*} \Delta^{l, s}$ equals that of $\Delta^{l, s}$. Using (3.6) we then have

$$
\begin{aligned}
\operatorname{dim} \Delta^{l, s} & =\operatorname{dim}\{l, s\}-\operatorname{dim}\left\{l-\frac{1}{2}, s-\frac{1}{2}\right\}= \\
& =(2 l+1)(2 s+1)-4 l s=2(l+s)+1 .
\end{aligned}
$$

We already know that $\Delta^{l, s}$ is an invariant subspace. From (3.7) and (3.9) we conclude that $\Delta^{l, s}$ spans the I.R. $D^{l+s}$; from (3.6) we then have

$$
D^{l} \otimes D^{s}=D^{l+s}+D^{l+s-1}+\ldots+D^{l-s} .
$$

A not yet normalized vector basis for $A^{{ }^{*}} \Delta^{l, s}$, for which $M$ is diagonal, is:

$$
\begin{aligned}
|r, t, l, s\rangle & \left.\equiv A^{\star} L_{-}^{t} a_{1}^{\star 2} 2 l b_{1}^{\star 2} 2 s\right\rangle, \quad t=0,1, \ldots 2(l+s) \\
M|r, t, l, s\rangle & =(l+s-t)|r, t, l, s\rangle .
\end{aligned}
$$

This is a consequence of the commutation relation (1.7). The normalization of (3.11) is easily found:

$$
\begin{aligned}
\langle 0, t, l, s \mid 0, t, l, s\rangle & =\left\langle 0, t-1, l, s\left|L_{+} L_{-}\right| 0, t-1, l, s\right\rangle= \\
& =t(2 l+2 s-t+1)\langle 0, t-1, l, s \mid 0, t-1, l, s\rangle
\end{aligned}
$$

where we used: $L_{+} L_{-}=L^{2}-M(M-1)$. By iteration and with the help of (3.8), we obtain:

$$
\langle r, t, l, s \mid r, t, l, s\rangle=\frac{r ! t !(2 l) !(2 s) !(2 l+2 s+r+1) !}{(2 l+2 s-t) !(2 l+2 s+1) !} .
$$

We now calculate the Clebsch-Gordan coefficient

$$
\left\langle l, m ; s, \mu \mid l, s, j, j_{3}\right\rangle,
$$

where we use the normalized vectors (see (2.5) and (3.11))

$$
\begin{aligned}
|l, m\rangle & =\frac{1}{c_{1}} a_{1}^{\star_{1} l+m} a_{2}^{\star} l-m|0\rangle \\
|s, \mu\rangle & =\frac{1}{c_{2}} b_{1}^{\star_{s}+\mu} b_{2}^{\varepsilon_{g}-\mu}|0\rangle \\
\left|l, s, j, j_{3}\right\rangle & =\frac{1}{c_{3}} L_{-}^{j-j_{3}} A^{\star} r a_{1}^{\star} p_{1}^{*}(2 j-p)|0\rangle .
\end{aligned}
$$


with

$$
\begin{aligned}
& c_{1}=\sqrt{(l+m) !(l-m) !} \\
& c_{2}=\sqrt{(s+\mu) !(s-\mu) !} \\
& c_{3} \text { given by }(2.16) .
\end{aligned}
$$

The relation between $j, p$ and $l, s$ will be given below (3.16). For the ClebschGordan coefficient we get:

$$
\begin{aligned}
& \left\langle l, m, s, \mu \mid l, s, j, j_{3}\right\rangle=\sum_{i=0}^{j-j_{3}} \sum_{k=0}^{r}\left(\begin{array}{c}
j-j_{3} \\
i
\end{array}\right)\left(\begin{array}{c}
r \\
k
\end{array}\right)(-1)^{r-k} \cdot \frac{1}{c_{1} c_{2} c_{3}} . \\
& \cdot\left\langle 0\left|a_{1}^{1+m} a_{2}^{l-m} b_{1}^{s+\mu} b_{2}^{s-\mu}\left(a_{2}^{*} a_{1}\right)^{i}\left(b_{2}^{*} b_{1}\right)^{j-j_{3}-i}\left(a_{1}^{*} b_{2}^{\star}\right)^{k}\left(a_{2}^{*} b_{1}^{*}\right)^{r-k} a_{1}^{*} b_{1}^{\star 2 j-p}\right| 0\right\rangle .
\end{aligned}
$$

Evaluation of the matrix element yields four Kronecker delta's, which are equivalent with

$$
p=j+l-s \quad r=-j+l+s
$$

and

$$
\delta_{k,-j+s+m+i} \delta_{j_{s}, m+\mu} .
$$

The first Kronecker delta is used to carry out the summation over $k$, the second is the final over-all delta. We obtain

$$
\begin{array}{r}
\left\langle l, m ; s, \mu \mid l, s, j, j_{3}\right\rangle=\sum_{i=0}^{j-j_{3}}\left(\begin{array}{c}
j-j_{3} \\
i
\end{array}\right)\left(\begin{array}{c}
-j+l+s \\
-j+s+m+i
\end{array}\right) \frac{(-1)^{l+m+i}}{c_{1} c_{2} c_{3}} . \\
\cdot(l+m+i) !(l-m) !(j+s-m-i) !(s-\mu) ! \delta_{j_{3}, m+\mu}
\end{array}
$$

Substituting (3.15) we get after some rearranging

$$
\begin{aligned}
\left\langle l, m ; s, \mu \mid l, s, j, j_{3}\right\rangle=\delta_{j_{3}, m+\mu} \cdot \\
\quad \cdot\left[\frac{(2 j+1)(l+s-j) !(l-m) !(s-\mu) !\left(j+j_{3}\right) !\left(j-j_{3}\right) !}{(j+l+s+1) !(l-s+j) !(-l+s+j) !(l+m) !(s+\mu) !}\right]^{\frac{1}{k}} . \\
\cdot \sum_{i=0}^{j-j_{3}}(-1)^{l+m+i} \frac{(l+m+i) !(j+s-m-i) !}{i !(l-m-i) !\left(j-j_{3}-i\right) !(s-j+m+i) !} .
\end{aligned}
$$

This is the same formula as obtained by Edmonds ${ }^{6}$ ) and is in agreement with the Condon and Shortley convention. The summation includes only the terms for which $(l-m-i)$ and $(s-j+m+i)$ are not negative.

4. Representations of $\mathrm{SU}_{3}$. To find the irreducible representations of $S U_{3}$ in an explicit and normalized realization we introduce the bose operators $a_{i}, b_{j}$, with $i, j=1,2,3$, and their adjoints. They satisty the usual commutation rules, and operate in the Hilbert space $Z$ for which an ortho- 
normal basis is given by

$$
\left|\begin{array}{c}
n_{1} n_{2} n_{3} \\
m_{1} m_{2} m_{3}
\end{array}\right\rangle=\prod_{i, j} \frac{a_{i}^{* m_{i}} b_{j}^{* n_{j}}}{\sqrt{m_{i} ! \cdot n_{j} !}}|0\rangle .
$$

We have for example

$$
b_{1}^{*} b_{1}\left|\begin{array}{c}
n_{1} n_{2} n_{3} \\
m_{1} m_{2} m_{3}
\end{array}\right\rangle=n_{1}\left|\begin{array}{c}
n_{1} n_{2} n_{3} \\
m_{1} m_{2} m_{3}
\end{array}\right\rangle .
$$

We define a representation of $S U_{3}$ in $Z$ by (see (1.14))

$$
A_{\mu v}=a_{\mu}^{*} a_{\nu}-b_{v}^{*} b_{\mu}-\frac{1}{3} \delta_{\mu \nu}(P-Q)
$$

where $P \equiv a_{\mu}^{*} a_{\mu}$ and $Q=b_{\mu}^{*} b_{\mu}$ (summationconvention). Eq. (4.1) yields a representation of $S U_{3}$ because (1.15) and the indeed commutation relations (1.16) are satisfied. Using (1.14) we find the representation for the generators, e.g.

$$
\begin{aligned}
& L_{+}^{1}=a_{1}^{*} a_{2}-b_{2}^{*} b_{1} \\
& M=\frac{1}{2}\left(a_{1}^{*} a_{1}-a_{2}^{*} a_{2}-b_{1}^{*} b_{1}+b_{2}^{*} b_{2}\right) \\
& Y=\frac{1}{3}\left(a_{1}^{*} a_{1}+a_{2}^{*} a_{2}-2 a_{3}^{*} a_{3}-b_{1}^{\star} b_{1}-b_{2}^{*} b_{2}+2 b_{3}^{*} b_{3}\right) .
\end{aligned}
$$

We next introduce $R^{*}=a_{\mu}^{*} b_{\mu}^{*}$ which commute with $A_{i j}$ and therefore with the group.

The representation of $S U_{3}$ in $Z$ reduced in much the same way as that of $S U_{2}$ in $X \otimes Y$.

a) The subspace $\{p, q\}$ defined by $P \psi=p \psi$ and $Q \psi=q \psi$ is invariant.

Dimension $\frac{1}{4}(p+1)(p+2)(q+1)(q+2)$

b) $\{p, q\}$ contains $R^{*}\{p-1, q-1\}$; orthoplement $\Delta_{p}^{q}$. An equivalent definition of $\Lambda_{p}^{q}$ is: the subspace of $\{p, q\}$ where the operator $R$ is identically zero: cf. section 3 where the same has been proved (with respect to $\left.S U_{2}\right)$ for $\Delta^{l, s}$. Splitting $\{p-n, q-n\}$ in the same way, and supposing $p \geqslant q$, we find (cf. (3.6))

$$
\{p, q\}=\sum_{n=0}^{q} R^{*} n_{p-n}^{q-n} .
$$

$\Lambda_{p}^{q}$ is an invariant subspace, and so are all terms in (4.3), because $R$ and $R^{*}$ commutc with the group (cf. section 3). We calculate the dimension of $A_{p}^{q}$ in the same way as in section 3 :

$$
\begin{aligned}
{\left[R, R^{*} n\right] } & =R^{* n-1} n(P+Q+n+2) \\
\left(R^{*} n_{\varphi_{1}}, R^{*} n_{\varphi_{2}}\right) & =n ! \frac{(p+q+n+2) !}{(p+q+2) !}\left(\varphi_{1}, \varphi_{2}\right)
\end{aligned}
$$

for $\varphi_{1}, \varphi_{\mathbf{2}} \varepsilon \Delta_{q}^{p}$. We thus have: $\operatorname{dim} R^{*} \Delta_{p}^{q}=\operatorname{dim} \Delta_{p}^{q}$, and, with (4.3)

$$
\operatorname{dim} \Lambda_{p}^{q}=\frac{1}{2}(p+1)(q+1)(p+q+2) .
$$


We already know that $\Delta_{p}^{q}$ is invariant (it spans a representation of the group). We will show it to be minimal invariant by generating the whole $\Delta_{p}^{q}$ out of one element with the aid of group elements. (Minimal invariant means that $\Delta_{p}^{q}$ has no trivial invariant subspace, and implies that it spans an I.R.)

We look for an element $|p q\rangle$ of $\Delta_{p}^{q}$, on which $L^{2}=L_{+}^{1} L_{-}^{1}+M(M-1)$ gives zero. Defining

$$
\begin{aligned}
T_{12}^{*} & =a_{1}^{*} b_{1}^{*}+a_{2}^{*} b_{2}^{*} \\
T_{3}^{*} & =a_{3}^{*} b_{3}^{*}
\end{aligned}
$$

and still supposing $p \geqslant q$, we put

$$
|p, q\rangle=\sum_{s=0}^{q} c_{s}^{(p, q)} T_{3}^{*} T_{12}^{* q-s} a_{3}^{* p-q}|0\rangle .
$$

This obviously is an element of $\{p, q\}$, and $L^{2}|p q\rangle=0$ because $L^{2}$ commutes with $a_{3}^{*}, b_{3}^{*}, T_{3}^{*}$ (trivial) and with $T_{12}^{*}$ :

$$
\left[L^{2}, T_{12}^{*}\right]=\left[L^{2},\left(T_{12}^{*}+T_{3}^{*}\right)\right]=\left[L^{2}, R^{\star}\right]=0 .
$$

We now must choose the $c_{s}^{(p, q)}$, such that $|p q\rangle$ is an element of the subspace $\Delta_{p}^{q}$ of $\{p, q\}$, that is such that $R|p q\rangle=0$. This equation yields a recurrence relation between $c_{s}$ and $c_{s+1}$ which is satisfied if we take

$$
c_{s}^{(p, q)}=(-1)^{s}\left(\begin{array}{l}
p \\
q
\end{array}\right)^{-1}\left(\begin{array}{c}
q+1 \\
s
\end{array}\right)\left(\begin{array}{c}
p \\
q-s
\end{array}\right) .
$$

Eq. (4.8) and (4.9) give $|p q\rangle$ in case $p \geqslant q$. If $p<q$ we have to interchange $p \leftrightarrow q$ and $a_{3}^{*} \leftrightarrow b_{3}^{*}$. In both cases $|p q\rangle$ is eigenvector of $M$ and $Y$, with eigenvalues 0 and $\frac{2}{3}(q-p)$, respectively.

$|p q\rangle$ is the element of $\Delta_{p}^{q}$ from which we will generate $\Delta_{p}^{q}$ in order to show that it is minimal invariant. We introduce:

$$
\left|x_{1} x_{2} x_{3} p q\right\rangle=\left(L_{+}^{1}\right)^{x_{1}}\left(L_{+}^{2}\right)^{x_{2}}\left(L_{+}^{3}\right)^{x_{3}}|p q\rangle . \quad x_{i} \text { integer } \geqslant 0 .
$$

We make the following statements

a) $\left|x_{1} x_{2} x_{3} p q\right\rangle$ is an clement of $\Delta_{p}^{q}$

b) the vectors (4.10) are orthogonal

c) the number of nonvanishing vectors (4.10) equals the dimension of $\Delta_{p}^{q}$. Statement $a$ ) is trivial because $R$ commutes with $L_{+}^{i}$. Statement $b$ ) is proved by showing that the vectors (4.10) are eigenvectors of $M, L^{2}$ and $Y$, with different eigenvalues for different sets $\left(x_{1} x_{2} x_{3}\right)$. Using the commutation relation (1.11) and the known properties of $|p q\rangle$ we find

$$
\begin{aligned}
M\left|x_{1} \ldots q\right\rangle & =\left(-\frac{x_{2}+x_{3}}{2}+x_{1}\right)\left|x_{1} \ldots q\right\rangle \\
L^{2}\left|x_{1} \ldots q\right\rangle & =\frac{x_{2}+x_{3}}{2}\left(\frac{x_{2}+x_{3}}{2}+1\right)\left|x_{1} \ldots q\right\rangle \\
Y\left|x_{1} \ldots q\right\rangle & =\left(2 \frac{q-p}{3}+x_{2}-x_{3}\right)\left|x_{1} \ldots q\right\rangle .
\end{aligned}
$$


Statement $c$ is derived from the fact that

$\left.c_{1}\right)\left|x_{1} x_{2} x_{3} p q\right\rangle \neq 0$ for $x_{3} \leqslant q, x_{2} \leqslant p$ and $x_{1} \leqslant x_{2}+x_{3}$

$\left.c_{2}\right)\left|x_{1} x_{2} x_{3} p q\right\rangle=0$ in all other cases.

$c$ then follows by performing a simple summation (cf. (4.6)). $c_{1}$ and $c_{2}$ will now be proved. From (4.8) and $L_{+}^{3}=a_{3}^{*} a_{1}-b_{1}^{*} b_{3}, L_{+}^{2}=a_{2}^{*} a_{3}-b_{3}^{*} b_{2}$ we have (take again $p \geqslant q$ ).

$$
\begin{aligned}
\left(L_{+}^{3}\right)^{q}|p q\rangle & =\zeta a_{3}^{*} p b_{1}^{*} q|0\rangle & \text { with } & \zeta \neq 0 \\
\left(L_{+}^{2}\right)^{p}\left(L_{+}^{3}\right)|p q\rangle & =\xi a_{2}^{*} p b_{1}^{*} q|0\rangle & \text { with } & \xi \neq 0
\end{aligned}
$$

where $\zeta$ and $\xi$ are numerical constants. Further we note that (use (1.11) and $L_{-}^{\mathbf{1}}|p q\rangle=0$ )

$$
\left(L_{+}^{2}\right)^{x_{2}}\left(L_{+}^{3}\right)^{x_{3}}|p q\rangle=\left(L_{+}^{3}\right)^{x_{3}}\left(L_{+}^{2}\right)^{x_{2}}|p q\rangle .
$$

Statement $c_{1}$ (as far as $x_{2}$ and $x_{3}$ are concerned) can easily be proved with (4.13) and (4.14). Statement $c_{2}$ for $x_{2}$ and $x_{3}$ can be proved directly from (4.12), the corresponding formula for $L_{+}^{2}$, and (4.14). The proofs for $x_{1}$ can be given along the same lines. Now that the correctlness of statements $a, b$ and $c$ has been shown, we may conclude that $\Delta_{p}^{q}$ is indeed minimal invariant; the I.R. spanned by $\Delta_{p}^{q}$ we call $D_{p}^{q}$.

The formulas (4.10) and (4.11) apply for every realization of $D_{p}^{a}$. They give a basis for $D_{p}^{q}$ provided we known the form of $|p q\rangle$, i.e. the element of $D_{p}^{q}$ for which $L^{2}$ gives zero. The normalization has been carried out in the appendix: the relation between the norms of $\left|x_{1} x_{2} x_{3} p q\right\rangle$ and of $|p q\rangle$ is given there. Also the norm of $|p q\rangle$ in the realization (4.8), (4.9) has been calculated.

5. Some remarks. We have found irreducible representations $D_{p}^{q}$ with $p, q=0,1,2 \ldots$ We must rely on abstract group theory where has been proved that there exist no other I.R. of $S U_{\mathbf{3}}$. The same we did for $S U_{\mathbf{2}}$. Every I.R. has been seen (4.11) to contain only one isosinglet $\left(L^{2}=0\right)$. The eigenvalue diagram (with $M$ and $Y$ axes) for $D_{p}^{q}$ can be drawn as follows (fig. 1). Put $|p q\rangle$ in the point $\left(0, \frac{2}{3}(q-p)\right)$. Each $L^{2}-$ multiplet $\left(x_{2}+x_{3}\right.$ fixed) consists of the vectors $\left|x_{1} x_{2} x_{3} p q\right\rangle$ with $x_{1}=0, \ldots, x_{2}+x_{3}$. We obtain the lowest point $x_{1}=0$ of such a multiplet by applying translations $L_{+}^{2}$ and $L_{+}^{3}, x_{2}$ respectively $x_{3}$ times, as shown in fig. 2 . The "higher" points of the multiplet are obtained next by applying $\left(L_{+}^{1}\right)^{x_{1}}$. There is a multiplet for every pair of values $0 \leqslant x_{2} \leqslant p$ and $0 \leqslant x_{3} \leqslant q$, with

$$
L_{0}=\frac{x_{2}+x_{3}}{2} \text { and } Y=\frac{2}{3}(q-p)+x_{2}-x_{3} .
$$

This is the Okubo rule for the "content" of an I.R. ?) Fig. 1 shows the diagram for $D_{1}^{4}$. For other diagrams, see ref. 5 . 
We label the I.R.'s by $p$ and $q$, the eigenvalues of $P$ and $Q$. This applies, however, only to the realization $\Delta_{p}^{q}$ of $D_{p}^{q}$. In $R^{\star} n_{p}^{q}$ the operators $P$ and $Q$ have other values. The reason is that $P$ and $Q$ are not built up from operators of the group. The I.R.'s must be distinguished by the eigenvalues of the Casimir operators: all independent operators that can be constructed from

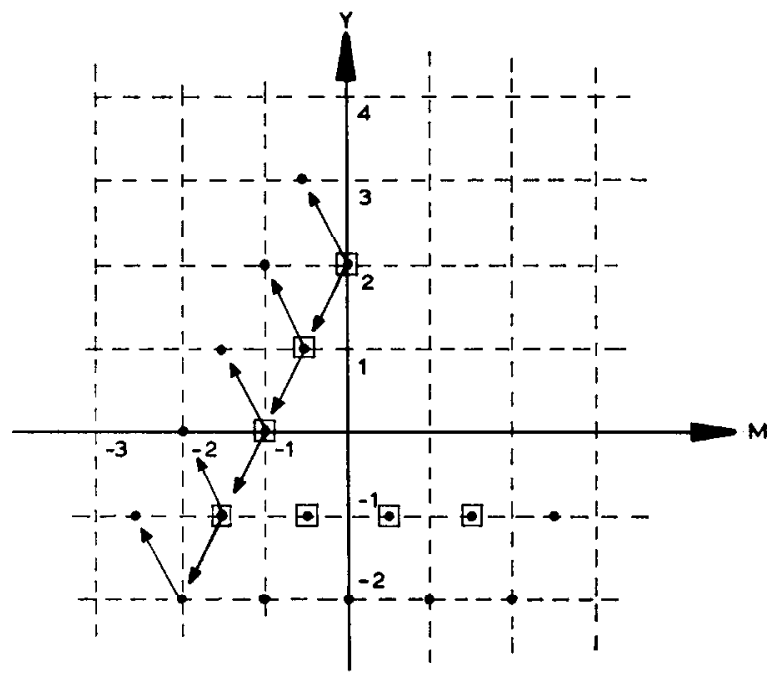

Fig. 1. The diagram for $D_{1}^{4}$. The lowest point of each isomultiplet is shown. Complete multiplets have been drawn for $Y=-1$ and $Y=-2$.

- multiplicity 1

$\square$ multiplicity 2 .

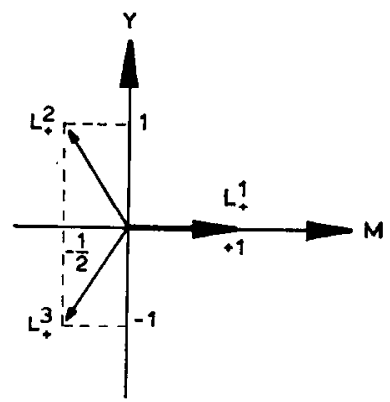

Fig. 2. The translational operators $L_{+}^{i}$.

group operators and commute with the group. As we should expect, there are two such operators for $S U_{3}$. They can be taken as

$$
\begin{aligned}
& F^{2}=\frac{1}{2} A_{\mu \nu} A_{\nu \mu} \\
& G^{3}=\frac{1}{2}\left(A_{\mu \nu} A_{\nu \lambda} A_{\lambda \mu}+A_{\mu \nu} A_{\lambda \mu} A_{\nu \lambda}\right) .
\end{aligned}
$$

In our representation $F^{2}$ becomes

$$
F^{2}=\frac{1}{3}(P+Q)(P+Q+3)-\frac{1}{3} P Q-R^{\star} R .
$$


$G^{3}$ distinguishes $D_{p}^{q}$ from $D_{q}^{p}$, between which $F^{2}$ makes no difterence. $F^{2}$ and $G^{3}$ commute with $R^{*}$ (tor $\left[R^{*}, A_{\mu \nu}\right]=0$ ), so $F^{2}$ and $G^{3}$ have the same value for $\Delta_{p}^{q}$ and $R^{\star} n \Delta_{p}^{q}$, which was not the case for $P$ and $Q$.

When we take (4.10) as basis for the I.R. $D_{p}^{q}$ we have fixed the relative phases within an I.R. It will be clear that within an isomultiplet the phases obey the Condon-Shortley convention: the matrix elements of $L_{+}^{\mathbf{1}}$ and $L_{-}^{1}$ are real and positive. The commonly adopted phase convention between different isomultiplets (all matrix elements of $L_{+}^{3}$ and $L_{-}^{3}$ positive) does not treat $L_{+}^{2}$ and $L_{+}^{3}$ on equal footing. We preferred the choice (4.10) which is entirely symmetric between $L_{+}^{2}$ and $L_{+}^{3}$ (cf. (4.14)), mainly because we did not intend to calculate Clebsch-Gordan coefficients. Only in simple cases $\left(D_{p}^{0} \otimes D_{\pi}^{0}\right.$ or $\left.D_{0}^{q} \otimes D_{0}^{k}\right)$ the reduction of product representations can be calculated in the same straightforward way as for $\mathrm{SU}_{2}$. In the general case one gets six operators like $R^{\star}$, which all commute with the product representation

$$
A_{\mu \nu}=a_{\mu}^{*} a_{v}+\alpha_{\mu}^{*} \alpha_{\nu}-b_{v}^{*} b_{\mu}-\beta_{\nu}^{*} \beta_{\mu}-\frac{1}{3} \delta_{\mu v}(P+\Pi-Q-K)
$$

of the group, and should all play a part in the reduction of this representation. Even if this reduction could be carried out, the resulting general expressions for the coupling coefficients (which then could be calculated in the same way as in section 3) would be clumsy and not very useful.

One of the authors (H.v. E.) has carried out this work as a staff member of the ,Stichting voor Fundamenteel Onderzoek der Materie" (F. O. M.).

\section{APPENDIX}

The normalization of the basisvectors for $D_{p}^{q}$. Take $\varphi=\left|0, x_{2}, x_{3}, p, q\right\rangle$ with $x_{2}=p-\alpha$ and $x_{3}=q-\beta$. We have

$$
2\left(\varphi, F^{2} \varphi\right)=\sum_{\mu \nu}\left(\varphi, A_{\mu \nu} A_{\nu \mu} \varphi\right) .
$$

Evaluating each term in the right hand side with the aid of (1.14) and (1.11), and using $L_{-}^{1} \varphi=0$, we get:

$$
\left(\varphi,\left[2 F^{2}-2 M^{2}-\frac{3}{2} Y^{2}+4 M\right] \varphi\right)=2\left(L_{+}^{2} \psi, L_{+}^{2} \psi\right)+2\left(L_{+}^{3} \varphi, L_{+}^{3} \varphi\right) .
$$

Using the notation $|\varphi|^{2}=|\alpha, \beta|^{2}$ we can write for (A.2)

$$
\begin{aligned}
|\alpha, \beta|^{2}(p \alpha+q \beta+\alpha \beta-\alpha(\alpha-1)-\beta(\beta-1))=\mid \alpha- & 1,\left.\beta\right|^{2}+ \\
& +|\alpha, \beta-1|^{2}
\end{aligned}
$$

From this

$$
\frac{|\alpha, \beta|}{|0,0|}
$$


could be calculated directly for all $\alpha \leqslant p$ and $\beta \leqslant q$ (put $|\alpha, \beta|=0$ for $\alpha$ or $\beta$ negative). We prefer to solve the equation (A.3). Substitute

$$
|\alpha, \beta|^{2}=\frac{(p-\alpha) !(q-\beta) !}{\alpha ! \beta !} B_{\alpha \beta}
$$

which gives

$$
\alpha(p-\alpha+1)\left(B_{\alpha \beta}-B_{\alpha-1, \beta}\right)+\beta(q-\beta+1)\left(B_{\alpha \beta}-B_{\alpha, \beta-1}\right)+\alpha \beta B_{\alpha \beta}=0 .
$$

From the symmetry of this equation we expect

$$
\alpha\left(B_{\alpha \beta}-B_{\alpha-1, \beta}\right)=\beta\left(B_{\alpha \beta}-B_{\alpha, \beta-1}\right) .
$$

Using this, we find from (A.4)

$$
\begin{aligned}
& B_{\alpha-1, \beta}=\frac{p+q-\alpha+2}{p+q-\alpha-\beta+2} B_{\alpha \beta} \\
& B_{\alpha, \beta-1}=\frac{p+q-\beta+2}{p+q-\alpha-\beta+2} B_{\alpha \beta} .
\end{aligned}
$$

When we first iterate (A.6) unto $B_{p, \beta}$, and then (A.7) unto $B_{p, q}$, we obtain

$$
B_{\alpha \beta}=\frac{B_{p q}}{(p+1) !(q+1) !} \frac{(p+q-\alpha+1) !(p+q-\beta+1) !}{(p+q-\alpha-\beta+1) !} .
$$

$B_{\alpha, \beta}$ as given by (A.8) satisfies (A.5), as one easily verifies. Moreover, it satisfies (A.6) and (A.7), which then means that it is the solution of (A.4). Returning to the original notation, and noting that

$$
\left(\varphi,\left(L_{-}^{1}\right)^{x_{1}}\left(L_{+}^{1}\right)^{x_{1}} \varphi\right)=\frac{x_{1} !\left(x_{2}+x_{3}\right) !}{\left(x_{2}+x_{3}-x_{1}\right) !}(\varphi, \varphi)
$$

for $\varphi=\left|0, x_{2}, x_{3}, p, q\right\rangle$, which derives from (1.11a and $\left.b\right)$ and (4.10), we finally get:

$$
\begin{aligned}
\left\langle x_{1} x_{2} x_{3} p q \mid x_{1} x_{2} x_{3} p q\right\rangle= & \frac{1}{(p+1)(q+1)\left(x_{2}+x_{3}+1\right)} \frac{x_{1} ! x_{2} ! x_{3} !}{\left(x_{2}+x_{3}-x_{1}\right) !}+ \\
& +\frac{\left(p+x_{3}+1\right) !\left(q+x_{2}+1\right) !}{\left(p-x_{2}\right) !\left(q-x_{3}\right) !}\langle p q \mid p q\rangle .
\end{aligned}
$$

To obtain an explicit normalization for our realization (4.8), (4.10), we must calculate the norm of $|p q\rangle$ or of any other element of (4.10), e.g. (4.12). We obtain

$$
\langle p q \mid p q\rangle=\left(\begin{array}{c}
p \\
q
\end{array}\right)^{-2}(p+q+1) ! \frac{q+1}{p+1} \text { for } p \geqslant q
$$


where use has been made of the formula

$$
\zeta \equiv \sum_{s=0}^{q}(-1)^{s} c_{s}^{(p q)}=\left(\begin{array}{c}
p \\
q
\end{array}\right)^{-1}\left(\begin{array}{c}
p+q+1 \\
q
\end{array}\right) .
$$

For $p<q$ we have to interchange $p$ and $q$ in (A.10) en (A.11).

Received 14-10-65

\section{REFERENCES}

1) Schwinger, J., “On angular momentum", Technical Information Service, Oak Ridge, Tennessee NYO 3071 (1952).

2) Moshinsky, M., Rev. mod. Phys. 34 (1962) 813.

3) Baird, G. E. and Biedenharn, L. C., J. math. Phys. 12 (1963) 1449.

4) Bargin an n, V., Comm. pure appl. Math., 14 (1961) 187; Rev. mod. Phys. 34 (1962) 829.

5) De Swart, J. J., Rev. mod. Phys. 35 (1965) 916.

6) Edmonds, A. R., "Angular momentum in quantum mechanics", Princeton University Press. (1957).

7) Okubo, S., Progr. theor. Phys. 27 (1962) 949. 